\title{
Contraposição da Equação de Froehlich com o tempo de simulação hipotética de rompimento recomendada da barragem de Jazigo, no sertão pernambucano
}

\author{
Counterposition of the Froehlich Equation with the hypothetical simulation time of recommended \\ breakdown of the Jazigo dam, in the sertão pernambucano \\ Contraposición de la Ecuación de Froehlich con el tiempo de simulación hipotética de rotura \\ recomendada de la presa Jazigo, en el sertão pernambucano
}

\author{
Alexson Caetano da Silva \\ ORCID: https://orcid.org/0000-0001-8253-7378 \\ Universidade Federal de Pernambuco, Brasil \\ E-mail: alexsoncaetano@hotmail.com \\ Natalia Fernanda Jeronimo de Santana \\ ORCID: https://orcid.org/0000-0003-0828-3204 \\ Universidade de Pernambuco, Brasil \\ E-mail: nfjs@poli.br \\ Gastão Cerquinha da Fonseca Neto \\ ORCID: https://orcid.org/0000-0003-3967-980X \\ Universidade Federal de Pernambuco, Brasil \\ E-mail: gastaocerquinha@gmail.com \\ Anna Elis Paz Soares \\ ORCID: https://orcid.org/0000-0001-9839-3057 \\ Universidade Federal de Pernambuco, Brasil \\ E-mail: anna.elis@ufpe.br \\ Amanda Machado Pereira \\ ORCID: https://orcid.org/0000-0002-4511-3522 \\ Universidade de Pernambuco, Brasil \\ E-mail: amp@poli.br \\ Simone Rosa da Silva \\ ORCID: https://orcid.org/0000-0001-7138-7546 \\ Universidade de Pernambuco, Brasil \\ E-mail: simonerosa@poli.br \\ Suzana Maria Gico Lima Montenegro \\ ORCID: https://orcid.org/0000-0002-2520-5761 \\ Universidade Federal de Pernambuco, Brasil \\ E-mail: suzanam.ufpe@gmail.com \\ José Roberto Gonçalves de Azevedo \\ ORCID: https://orcid.org/0000-0002-2662-7341 \\ Universidade Federal de Pernambuco, Brasil \\ E-mail: jose.azevedo@ufpe.br
}

\begin{abstract}
Resumo
O presente estudo tem como objetivo apresentar uma análise sobre a mancha de inundação causada pela ruptura hipotética da barragem de Jazigo nos tempos de formação de brecha de 0,10h, 0,30h, recomendados pela Agência Nacional de Águas e Saneamento Básico (ANA) e o tempo de formação de brecha de 2,15h, calculado através da equação de Froelich. A estruturação da metodologia consistiu na caracterização da barragem; elaboração do hidrograma de ruptura através de software HEC-HMS 4.3; realização da simulação do rompimento com o software HEC-RAS 6.0 Beta para os três tempos de formação de brecha; elaboração dos mapas de inundação de profundidade, velocidade e intensidade, com a classificação dos níveis de alerta; e por fim, a análise de três locais do município de Serra Talhada-PE, situado a $7 \mathrm{~km}$ da estrutura da barragem. Concluiu-se que, para os três diferentes tempos de formação, o indicador de intensidade está no nível de alerta alto. Além disso, os indicadores de profundidade e velocidade apresentaram mapas semelhantes. Nas três seções de referência do município, a velocidade da onda de cheia divergiu de forma mais abrupta apenas no tempo de formação de brecha de 2,15h, mantendo-se a altura do nível d'água similar entre os tempos. Por fim, espera-se contribuir para a elaboração do Plano de Ação Emergencial da barragem, para que com as ações de mitigação possam diminuir os riscos da população do município em uma possível ruptura.
\end{abstract}

Palavras-chave: Segurança de barragem; Mancha de inundação; Simulação de rompimento. 


\begin{abstract}
The present study aims to present an analysis of the flood spot caused by the hypothetical rupture of the Jazigo dam at the times of breccia formation of $0.10 \mathrm{~h}, 0.30 \mathrm{~h}$, recommended by the National Agency for Water and Basic Sanitation (ANA) and the gap formation time of $2.15 \mathrm{~h}$, calculated using the Froelich equation. The structuring of the methodology consisted of the characterization of the dam; elaboration of the rupture hydrograph using HEC-HMS 4.3 software; performing the rupture simulation with the HEC-RAS 6.0 Beta software for the three gap formation times; elaboration of inundation maps of depth, velocity and intensity, with classification of alert levels; and finally, the analysis of three sites in the municipality of Serra Talhada-PE, located $7 \mathrm{~km}$ from the dam structure. It was concluded that, for the three different training times, the intensity indicator is at the high alert level. Furthermore, the depth and velocity indicators had similar maps. In the three reference sections of the municipality, the flood wave velocity diverged more abruptly only at the time of breccia formation of $2.15 \mathrm{~h}$, with the height of the water level being similar between the times. Finally, it is expected to contribute to the preparation of the Emergency Action Plan for the dam, so that mitigation actions can reduce the risks of the municipality's population in a possible rupture.
\end{abstract}

Keywords: Dam safety; Flood stain; Breakthrough simulation.

\title{
Resumen
}

El presente estudio tiene como objetivo presentar un análisis del punto de inundación provocado por la hipotética rotura de la presa de Jazigo en los momentos de formación de brechas de 0,10h, 0,30h, recomendado por la Agencia Nacional de Agua y Saneamiento Básico (ANA) y la brecha tiempo de formación de 2,15h, calculado mediante la ecuación de Froelich. La estructuración de la metodología consistió en la caracterización de la presa; elaboración del hidrograma de ruptura utilizando el software HEC-HMS 4.3; realizar la simulación de ruptura con el software HECRAS 6.0 Beta para los tres tiempos de formación de huecos; elaboración de mapas de inundaciones de profundidad, velocidad e intensidad, con clasificación de niveles de alerta; y finalmente, el análisis de tres sitios en el municipio de Serra Talhada-PE, ubicado a $7 \mathrm{~km}$ de la estructura de la presa. Se concluyó que, para los tres tiempos de entrenamiento diferentes, el indicador de intensidad se encuentra en el nivel de alerta alto. Además, los indicadores de profundidad y velocidad tenían mapas similares. En las tres secciones de referencia del municipio, la velocidad de la ola de crecida divergió más abruptamente solo en el momento de la formación de la brecha de 2,15h, siendo la altura del nivel del agua similar entre los tiempos. Finalmente, se espera contribuir a la elaboración del Plan de Acción de Emergencia de la presa, para que las acciones de mitigación puedan reducir los riesgos de la población del municipio en una posible ruptura.

Palabras clave: Seguridad de presas; Mancha de inundación; Simulación revolucionaria.

\section{Introdução}

As barragens trazem vantagens e são essenciais para a sociedade, contudo em razão de serem obras de grande dimensão, por conterem grandes volumes e em virtude das características físico-químicas específicas dos materiais armazenados, suas falhas podem causar destruição e levar a perdas de vidas humanas e materiais (Souza, 2019). De acordo com Sabau e Şerban (2018) o colapso de uma barragem é o rompimento ou deslocamento de uma parte do corpo da barragem ou de sua fundação, o barramento perde a sua função de contenção, gerando a liberação descontrolada de um grande volume de água do reservatório em curto período. A ruptura pode acontecer por motivos diversos que variam desde infiltração, erosão interna, galgamento, liquidação devido a escorregamentos de taludes e liquefação devido a terremotos.

De acordo com o censo de 2019 da Comissão Internacional de Grandes Barragens - ICOLD (2019), existem mais de 58.000 barragens de grande dimensão espalhadas por todo o mundo, a maior parte construídas nos últimos 60 anos, devido ao aumento populacional que levou à expansão das demandas hídrica. No Brasil, o primeiro registro de um barramento construído data no fim do século XVI, localizado na cidade do Recife, atualmente é conhecido como “Açude Apipucos”(Mello, 2011). Segundo a Agência Nacional de Águas e Saneamento Básico - ANA, no final dos anos sessenta, estudos relacionados a segurança de barragens passaram a ter mais destaque, como a reavaliação das barragens e vertedouros mais antigos, os efeitos do envelhecimento delas e os impactos ambientais causados pelos barramentos. A partir de então, muitos países criaram suas próprias legislações de segurança de barragens, devido à ocorrência de acidentes e incidentes, por isso diverge entre países a abordagem das questões organizativas e técnicas (ANA, 2016). No Brasil, foi instituída a Lei N 12.334, nomeada como Política Nacional de Segurança de Barragens - PNSB em 2010 (Brasil, 2010). Tornando obrigatório que barragens acima de $15 \mathrm{~m}$ de altura do maciço, com capacidade total do reservatório de $3.000 .000 \mathrm{~m}^{3}$, reservatório que contenha resíduos perigosos 
conforme normas técnicas aplicáveis ou categoria de dano potencial associado médio ou alto, em termos econômicos, sociais, ambientais ou de perda de vidas humanas, devem obter seu Plano de Segurança de Barragem - PSB - e o Plano Ação Emergencial - PAE. Em setembro de 2020, a referida Lei foi atualizada pela Lei $\mathrm{N}^{\circ} 14.066$ (Brasil, 2020), onde alguns pontos relevantes sofreram alterações: a altura da barragem, que antes era considerado do maciço de fundação até a cota da crista e hoje deve-se considerar a altura a partir do pé do talude de jusante até a crista de coroamento do barramento; a definição de empreendedor, que pode ser uma pessoa física ou jurídica que detenha algum direito sobre o empreendimento ou o explore oficialmente; também, houve uma ampliação do conceito de dano potencial associado à barragem e a inclusão do PAE no PSB, entre outros tópicos.

A barragem de Jazigo, situada no município de Serra Talhada, no sertão pernambucano, foi caracterizada pelo seu empreendedor, a Secretaria de Infraestrutura e Recursos Hídricos de Pernambuco - SEINFRA-PE, como Nível de Perigo Global 2 (alerta), segundo a Inspeção de Segurança Regular de 2020 (SEINFRA, 2020). A barragem foi construída em alvenaria de pedra, e fica a apenas $7 \mathrm{~km}$ a montante do centro da cidade, de tal forma que seu possível rompimento causaria extensos danos à sede do município e sua população.

Nesse contexto, este estudo visa determinar o impacto nos mapas de inundações gerados pela variação nos tempos de formação das brechas de ruptura hipotética, recomendados pela ANA e calculado pela equação de Froehlich (Froehlich et al, 2016), através de estudo de caso realizado na barragem de Jazigo, situada no sertão de Pernambuco. Esses recursos são de extrema importância no planejamento e antecipação de ações por parte do poder público para a evacuação de pessoas e a gestão do risco em uma situação de iminência de rompimento.

\section{Metodologia}

Para desenvolvimento da pesquisa, foi aplicado o método científico de estudo de caso, empregando evidências quantitativas (Yin, 2015; Pereira et al, 2018) para avaliar o comportamento do rompimento hipotético da Barragem Jazigo, utilizando na simulação os tempos de ruptura de $0,10 \mathrm{~h}$ e $0,30 \mathrm{~h}$ recomendados pela ANA (ANA, 2016) e o tempo calculado pela equação de Froehlich, de 2,15h, observando as principais diferenças temporais e espaciais da profundidade do escoamento e dos mapas com indicadores e índices de perigo. As etapas da metodologia utilizada são detalhadas nos tópicos a seguir.

\section{1 Área de estudo}

A barragem de Jazigo pertence ao governo do Estado de Pernambuco, localizada a aproximadamente $405 \mathrm{~km}$ da cidade do Recife, está situada na cidade de Serra Talhada no estado de Pernambuco, nas coordenadas de 759'57,71"S e $38^{\circ} 14^{\prime} 30,76$ "W. Foi inaugurada no ano de 1983 com capacidade para armazenamento de $15.543 .300 \mathrm{~m}^{3}$, constituída de alvenaria de pedras e projetada com a finalidade de aumentar a oferta hídrica na região (SEINFRA, 2020).

O curso d'água barrado é o rio Pajeú, um dos principais rios do Estado, que constitui a maior bacia hidrográfica pernambucana. Dentre os 27 municípios que o rio Pajeú margeia está Serra Talhada (Rocha, 2018), com aproximadamente 86.915 habitantes segundo o Instituto Brasileiro de Geografia e Estatística - IBGE (2020). O município é uma das principais cidades do sertão de Pernambuco por ser polo em saúde, educação e comércio ～(JFPE, 2004).

A análise da sub-bacia da barragem foi gerada a partir do software ArcMap 10.5, com a base de dados da Missão Topográfica Radar Shuttle (SRTM), que fornece o raster do Modelo Digital de Elevação (MDE) através do Serviço Geológico dos Estados Unidos (USGS), conforme mostra a Figura 1. 
Figura 1. Mapa de localização da bacia hidrográfica da barragem de Jazigo.

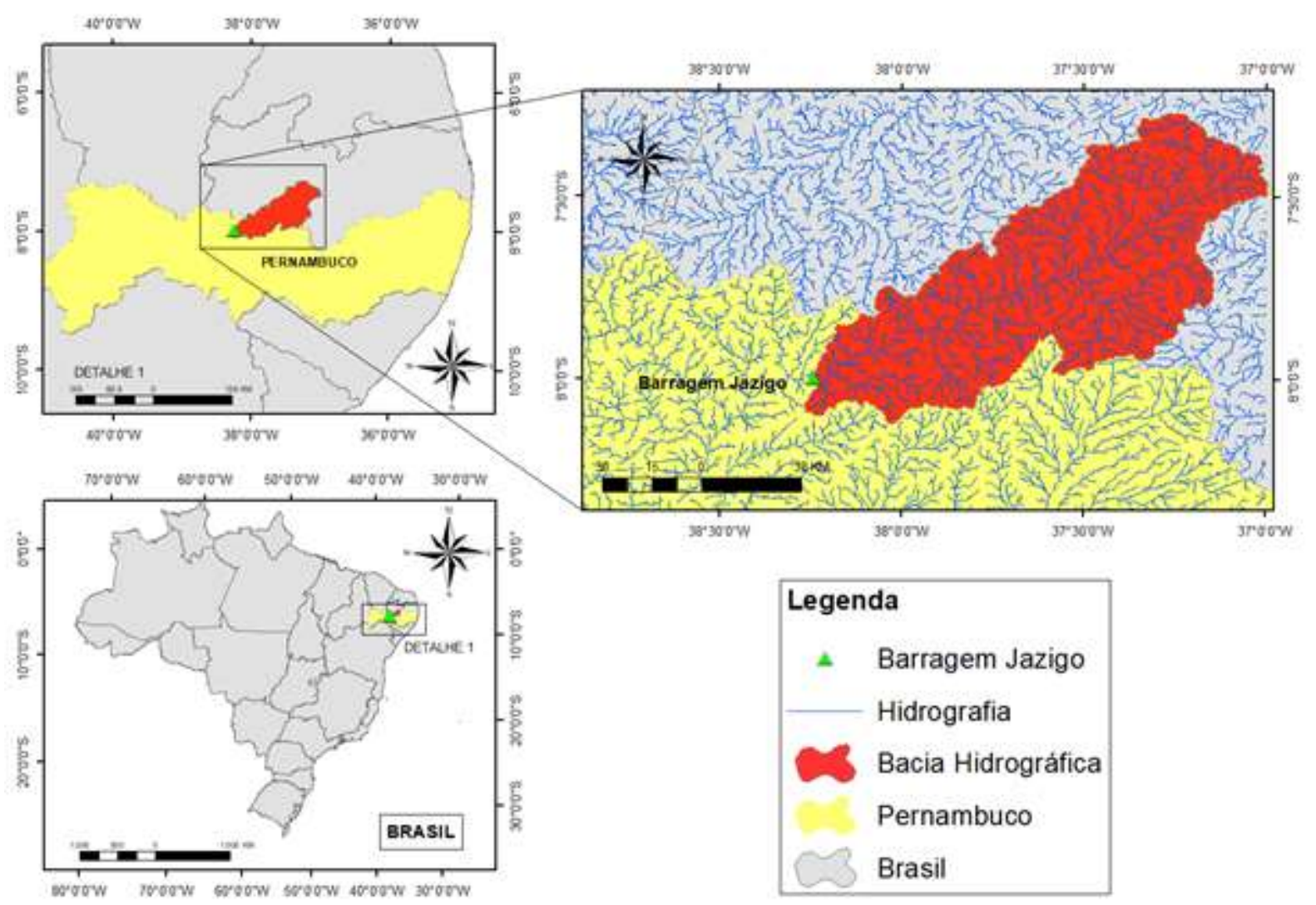

Fonte: Autores, a partir de dados do IBGE.

\subsection{Brecha de ruptura}

O desenvolvimento da brecha de ruptura é idealizado como um processo paramétrico, definido pela forma da brecha, seu tamanho final e o tempo necessário para seu desenvolvimento, frequentemente chamado de tempo de falha (Wahl, 2004).

Barragens podem romper de forma gradual ou instantaneamente. O tipo de ruptura depende, principalmente, da causa da falha e do tipo da barragem. Os principais mecanismos de rompimento são: ruptura da fundação overtopping ou galgamento, normalmente por um evento hidrológico extremo, e piping ou ruptura por entubamento, em função de falhas no corpo da barragem. (Faria et al, 2019).

Nas barragens de concreto de gravidade, considera-se que a área da brecha é igual a uma parte apreciável da área da seção transversal da barragem definida pelo eixo de referência, considerando a geometria da brecha como retangular. Já as barragens de aterro apresentam uma problemática da caracterização da brecha de ruptura que correspondem a rupturas parciais e lentas, sendo usualmente caracterizados alguns parâmetros, como: a forma das brechas, que é usualmente trapezoidal, e a altura da brecha, que pode ser considerada como aproximadamente igual à altura da barragem (ANA, 2016, p. 36-37).

\subsection{Tempo de ruptura}

Para este trabalho, foram utilizados os tempos de ruptura mínimo e máximo para barragens de gravidade em concreto indicados pela ANA (2016), apresentados na Tabela 1, correspondendo a 0,1 e 0,3 horas. Estabelecidos estes critérios, é possível verificar as principais diferenças nos mapas de inundação e como são determinantes em uma situação real. 
Tabela 1. Valores característicos da brecha de ruptura.

\begin{tabular}{|c|c|c|c|}
\hline Tipo de Barragem & $\begin{array}{l}\text { Largura média da brecha } \\
\left.\qquad \underline{B}_{b r e}\right)\end{array}$ & $\begin{array}{c}\text { Componente } \\
\text { horizontal da } \\
\text { inclinação da brecha } \\
(1 \mathrm{~V}: \mathrm{ZH})\end{array}$ & $\begin{array}{l}\text { Tempo de ruptura } \\
\text { (horas) }\end{array}$ \\
\hline Concreto em arco & $\begin{array}{l}\text { Todo o desenvolvimento da } \\
\text { barragem ou } \underline{B}_{\text {bre }} \geq 0,8_{B_{\text {barr }}}\end{array}$ & $\begin{array}{c}0 \leq Z_{\text {vale }} \\
\text { Inclinação do }\end{array}$ & $t_{\text {rot }} \leq 0,1$ \\
\hline Concreto gravidade & $\begin{array}{c}\text { Um múltiplo de vários blocos, } \\
\text { sendo usualmente, } \underline{B}_{\text {bre }} \leq \\
0,5_{B_{h a r r}}\end{array}$ & $\mathrm{Z}=0$ & $0,1 \leq t_{r o t} \leq 0,3$ \\
\hline Terra/ enrocamento & $H_{\text {barr }} \leq \underline{B}_{\text {bre }} \leq 5 H_{\text {barr }}$ & $1 / 4 \leq \mathrm{Z} \leq 1$ & $0,5 \leq t_{r o t} \leq^{3}$ \\
\hline Estéreis de minas & $\underline{B_{b r e}} \leq 0,8 H_{b a r r}$ & $1 \leq \mathrm{Z} \leq 2$ & $0,1 \leq t_{r n t} \leq 0,3$ \\
\hline
\end{tabular}

Fonte: Adaptado de ANA (2016, p. 37) e USBR (1989).

Ainda, foi calculado o tempo de ruptura através da equação de Froehlich (Equação 1), criada a partir do estudo de 63 casos de ruptura de barragens em 1995 e revisado no ano 2011, considerando 111 casos de ruptura de barragens (Froehlich et $a l, 2016)$. Segundo esses autores, as rupturas de barragens se desenvolvem de uma forma pressuposta, geralmente na forma de um trapézio que é definido por sua altura final, largura de base ou largura média e inclinações laterais, junto com o tempo necessário para a ruptura ocorrer completamente.

Onde:

$$
\text { Bave }=0,23 k_{0} V w^{1 / 3}
$$

Bave : largura média da brecha $(\mathrm{m})$;

$V w$ : volume do reservatório no momento da ruptura $\left(\mathrm{m}^{3}\right)$;

$k_{0}$ : constante equivalente a 1,5 para casos de rompimento por galgamento.

\subsection{Cenários de Ruptura}

Conceitua-se cenário como uma combinação única de circunstâncias com interesse para uma avaliação de riscos de um determinado ambiente, viabilizando o estudo e auxiliando na representação das situações potencialmente mais graves (Dias, 2011). Devido à dificuldade para se determinar a abertura da brecha e a caracterização das barragens, mais de um cenário de ruptura é considerado.

Deve-se optar por construir o menor número possível de cenários. Assim, para garantir uma adequada segurança associada aos diferentes tipos de barragens, devem ser definidos os seguintes cenários no PAE: o cenário de operação hidráulica extrema, que sem conduzir à ruptura pode dar origem a descargas importantes e, de igual forma, colocar em risco pessoas e bens no vale a jusante; e o cenário de ruptura propriamente dita (ANA, 2016, p. 38).

Segundo o Corpo de Engenheiros do Exército dos Estados Unidos - USACE (2014), a metodologia utilizada estabelece três tipos de cenários: dia ensolarado com rompimento da barragem, inundação com rompimento da barragem, cheias sem rompimento da barragem (inundação sem rompimento).

Para o cenário da ruptura da barragem Jazigo foi considerado um dia de sol, com uma chuva de projeto com tempo de retorno de 100 anos. O cenário considerado foi o de colapso estrutural após sobrecarga hidráulica, sendo o vertedouro obstruído com uma lâmina d'água na cota $85,5 \mathrm{~m}$. 


\subsection{Hidrograma de ruptura}

O hidrograma de ruptura foi desenvolvido através da curva cota área volume da bacia hidráulica da barragem Jazigo, conforme Figura 2, dos parâmetros de formação de brecha pré-estabelecidos e da curva intensidade-duração-frequência (IDF) do posto pluviométrico de Triunfo, caracterizado por Silva et al (2009), sendo o posto mais próximo da barragem já estudado. Para geração do hidrograma, foi utilizado o software HEC-HMS 4.3, programa desenvolvido pela USACE que calcula a precipitação por meio do coeficiente de Runoff.

Figura 2. Curva cota-área-volume da Barragem Jazigo.

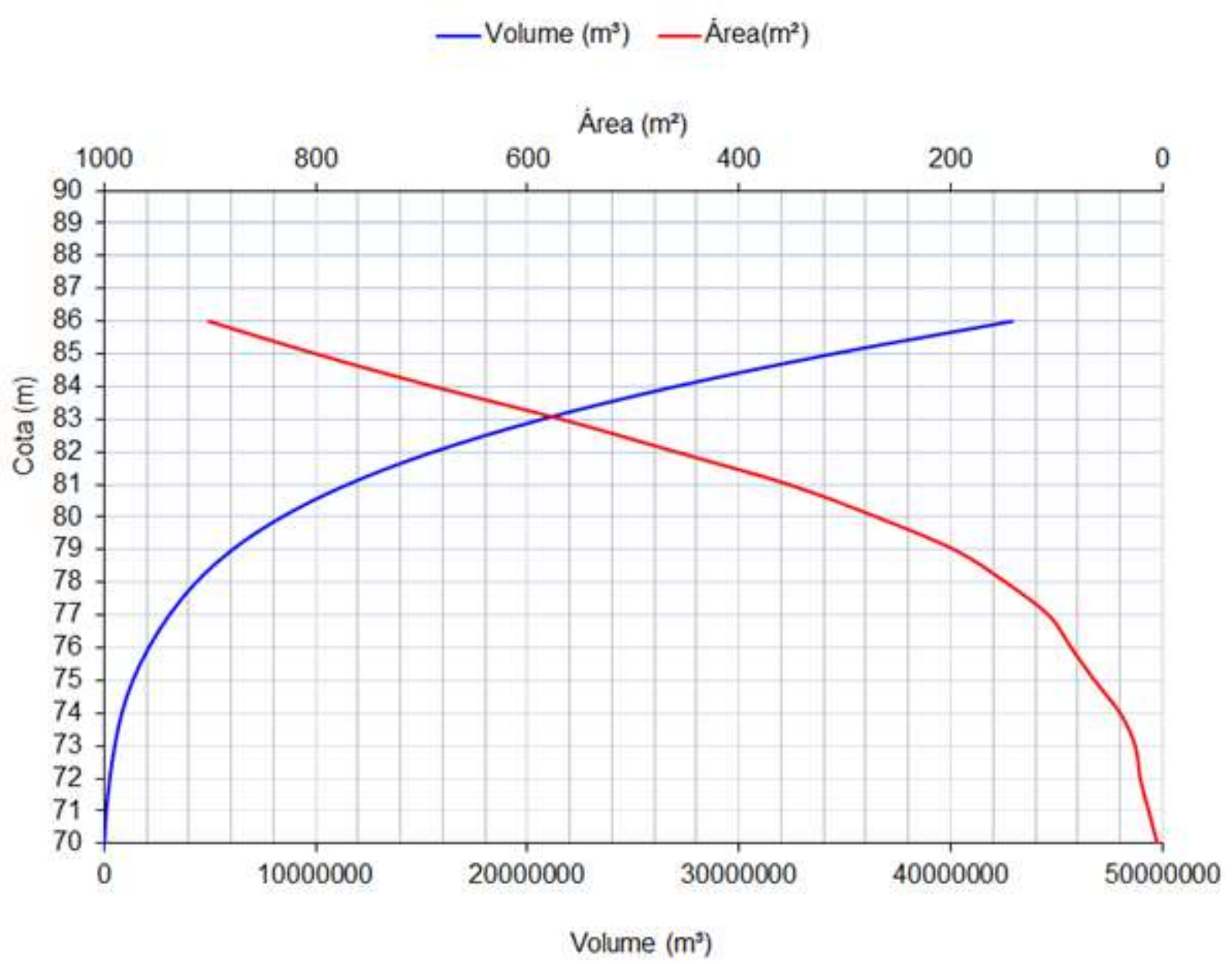

Fonte: Autores, a partir de dados da apac.

\subsection{Modelos hidrodinâmicos}

"Para efetuar a simulação do processo de formação de brechas, podem ser utilizados modelos paramétricos, ou seja, modelos em que é exigido ao modelador a definição da geometria da brecha (a largura e a forma final) e o tempo de ruptura" (ANA, 2016. p. 34). O software HEC- RAS 6.0 Beta foi criado pelo corpo de engenheiros do exército dos Estados Unidos, funcionando através de cálculos hidráulicos em uma e duas dimensões (1 e 2 D) e acoplado 1D/2D, com regime de escoamento permanente, quase não permanente e não permanente (Neves, Rodrigues e Cabral, 2021).

A metodologia do modelo de ruptura se baseia em: pré-processamento de dados geométricos para software HEC-RAS usando HEC-GeoRAS, análise hidráulica em HEC-RAS e pós-processamento dos resultados do HEC-RAS e mapeamento da planície de inundação usando o HEC-GeoRAS (Balogun E Ganiyu, 2017).

Para a modelagem hidrodinâmica foi necessário todo o estudo de caracterização geométrica da barragem Jazigo, além 
do modelo digital de elevação - MDE, que é a representação de elementos presentes na superfície terrestre, por meio do estudo topográfico, modelos em 3D e curvas de níveis. A base topográfica utilizada foi a do Pernambuco Tridimensional - PE3D, o qual apresenta nível de precisão de 1:1.000. Ademais, foram necessárias informações do coeficiente de rugosidade, condições de contorno e condições de cálculo. A extensão modelada a jusante da bacia hidráulica foi adquirida segundo a Equação 2, válida nos limites inferiores a $6,7 \mathrm{~km}$ e superiores a $100,4 \mathrm{~km}$ de distância, para reservatórios entre 0 e $1.000 \mathrm{hm}^{3}$ (Banco Mundial, 2014).

$$
\operatorname{Dmax}=8,870 * 10^{-8} *(\operatorname{Vmax})^{3}-2,602 * 10^{-4} *(\operatorname{Vmax})^{2}+2,648 * 10^{-1} *(\operatorname{Vmax})+6,737
$$

Se $\operatorname{Vmax} \leq 1.000 \mathrm{hm}^{3}$.

Em que,

Vmax: volume máximo do reservatório $\left[\mathrm{hm}^{3}\right]$;

Dmax: distância máxima a jusante do reservatório [km].

O modelo hidrodinâmico bidimensional desenvolvido no HEC-RAS utiliza as equações de Navier-Stokes, em que é representado o movimento de um fluido viscoso e incompressível. As equações completas da Conservação da Massa e Conservação do Momento são conhecidas como Equações de Águas Rasas - EAR (Equações 3 e 4). Algumas simplificações nos termos das equações foram realizadas com o objetivo de reduzir o custo computacional nos processos hidráulicos, tais como modelos de onda difusa e onda cinemática. No entanto, as utilizações das EAR completas são essenciais na representação de regimes complexos, como escoamentos transcritos, supercríticos ou descontinuidades de escoamento de choque (Guo et al, 2021).

$$
\frac{\delta H}{\delta t}+\frac{\delta(h u)}{\delta x}+\frac{\delta(h v)}{\delta y}+q=0
$$

Equação da conservação da massa (3)

onde,

H: elevação da água [1];

h: profundidade da água no ponto [1];

t: tempo [T];

u e v: componentes da velocidade nas direções x e y, respectivamente [1/T];

q: termo de fluxo de entrada e saída do sistema [12/T].

$$
\begin{aligned}
\frac{\delta u}{\delta t}+ & u \frac{\delta u}{\delta x}+v \frac{\delta u}{\delta y}=-g \frac{\delta H}{\delta x}+v_{t}\left(\frac{\delta^{2} u}{\delta x^{2}}+\frac{\delta^{2} u}{\delta y^{2}}\right)-c_{f} u+f v \\
& \frac{\delta v}{\delta t}+u \frac{\delta v}{\delta x}+v \frac{\delta v}{\delta y}=-g \frac{\delta H}{\delta y}+v_{t}\left(\frac{\delta^{2} v}{\delta x^{2}}+\frac{\delta^{2} v}{\delta y^{2}}\right)-c_{f} v+f u
\end{aligned}
$$

onde,

u e v: velocidades nas direções cartesianas [1/T];

$\mathrm{g}$ : aceleração da gravidade $\left[1 / \mathrm{T}^{2}\right]$;

vt: coeficiente de viscosidade dinâmica $\left[1^{2 / T}\right]$;

cf: coeficiente de atrito $\left[\mathrm{m} / \mathrm{l} / \mathrm{T}^{2}\right]$;

f: coeficiente de Coriolis (Brunner, 2016). 


\subsection{Parâmetros para classificar o mapa de inundação}

O estudo das consequências do rompimento tem por objetivo a previsão do hidrograma de saída da barragem, a determinação das propriedades hidráulicas e a propagação espacial da inundação gerada (Pietro Calderon et al, 2017). No Brasil, de acordo com o Manual do Empreendedor sobre Segurança de Barragens (ANA, 2016), a definição da mancha de inundação deve ser feita preferencialmente sobre cartografia 1:25000, em zonas urbanas ou industriais pode ser completada com maior minúcia. Em relação aos perfis, devem ser traçados para diferentes seções transversais do rio, as seções devem encontrar de preferência com elementos de risco. É recomendado, durante os primeiros $5 \mathrm{~km}$ a jusante da barragem, que os resultados do cálculo do mapa de inundação sejam espaços de $1 \mathrm{~km}$, depois dos $5 \mathrm{~km}$ iniciais prossegue com uma sucessão regular de intervalos maiores, garantindo sempre informação mais detalhada nas zonas singulares.

Ainda de acordo com a ANA (2016, p.43), o mapa de inundação deve conter as seguintes informações:

- identificação do cenário que lhes corresponde;

- limites das zonas inundáveis;

- limites administrativos das áreas atingidas (estado, município, localidade);

- vias de comunicação inundadas e identificação das obras de arte atingidas;

- infraestruturas e instalações importantes ou existência de instalações de produção ou de armazenagem de substâncias perigosas.

Por meio do software de geoprocessamento ArcGis, foi possível obter os mapas de inundação da simulação de ruptura. Mediante a ferramenta Raster Calculator, foram elaborados mapas com objetivo de caracterizar a área de influência para diferentes profundidades, velocidades e intensidades. O produto da profundidade e da velocidade gera a intensidade. Em concordância com o estudo de Ribeiro Neto et al (2016) foram utilizados os limites de indicadores expostos na Tabela 2 para a construção dos mapas de perigo.

Tabela 2. Limites de indicadores para construir um mapa de perigo.

\begin{tabular}{|c|c|c|c|}
\hline Indicador & Baixo & Médio & Alto \\
\hline Profundidade (m) & $0-0,60$ & $0,60-1,20$ & $>1,20$ \\
\hline Velocidade (m/s) & $0-0,60$ & $0,60-1,20$ & $>1,50$ \\
\hline Intensidade $\left(\mathrm{m}^{2} / \mathrm{s}\right)$ & $0-0,36$ & $0,36-1,50$ & $>$ \\
\hline
\end{tabular}

Fonte: Ribeiro Neto et al (2016, p. 384)

A determinação das zonas mais vulneráveis é proporcionada por intermédio dessa classificação que colabora para estudos futuros.

\section{Resultados e Discussão}

A partir dos procedimentos descritos no item anterior, foi possível obter os mapas de profundidade, velocidade e intensidade da barragem de Jazigo para os três tempos de formação de brecha pré-estabelecidos, analisando as principais diferenças e semelhanças entre os resultados. Sendo inicialmente gerados os mapas do indicador de profundidade, seguindo os critérios de Ribeiro Neto et al (2016) (Figuras 3). 
Figura 3. Mapas de profundida para os tempos de formação de brecha. Tempo de formação de brecha de $0,10 \mathrm{~h}$ (A), tempo de formação de brecha de $0,30 \mathrm{~h}$ (B) e para o tempo de formação de brecha de $2,15 \mathrm{~h}$.
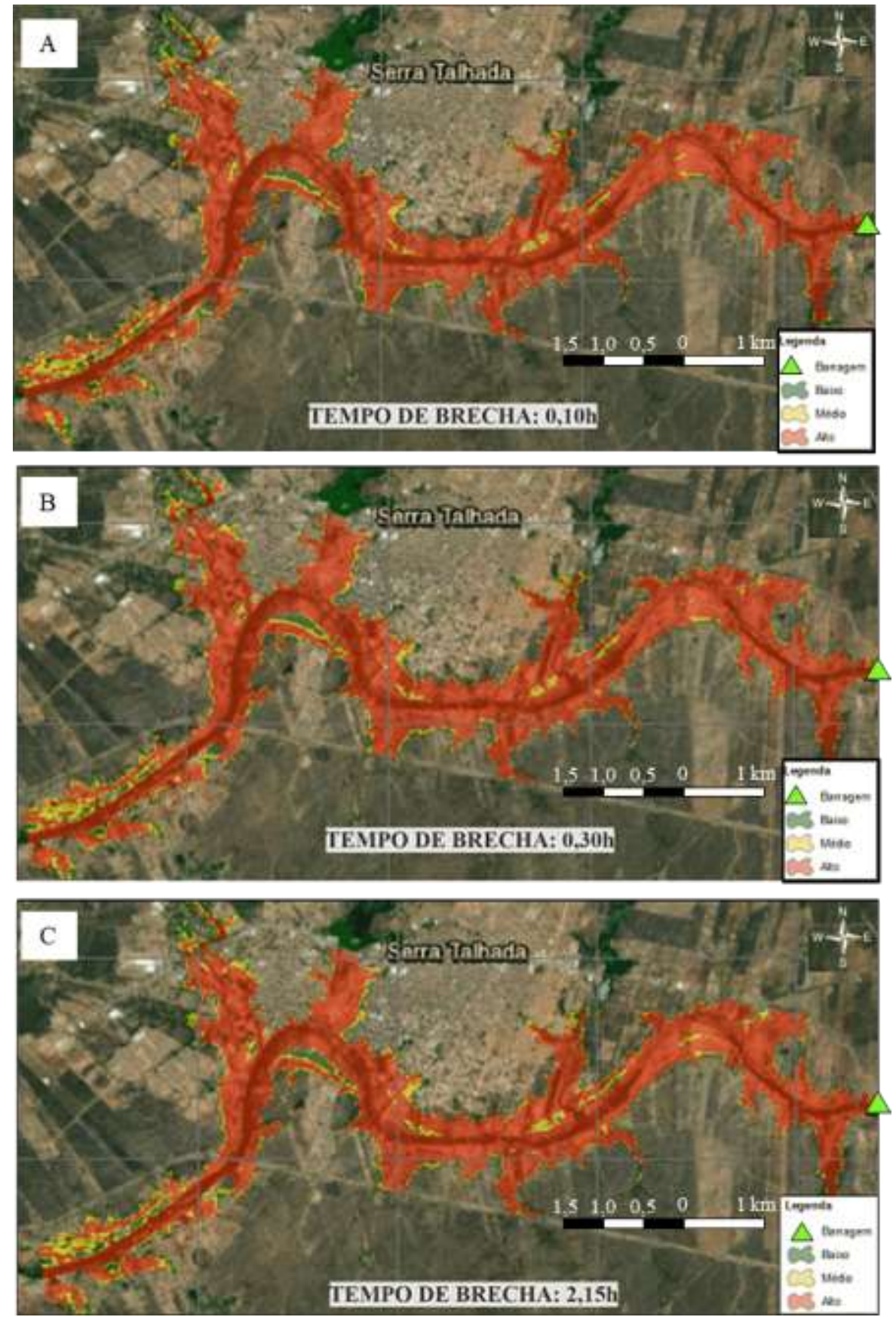

Fonte: Autores.

O indicador de profundidade, conforme Figura 3, nos três tempos analisados de formação de brecha, teve em sua grande parte o nível alto, sendo possível perceber uma leve mudança para o nível médio no final do trecho ensaiado. Entretanto, os picos de profundidade foram bastante distintos. O tempo de formação de brecha de $2,15 \mathrm{~h}$, obtido através da equação de Froehlich, apresentou o menor pico, de 4,98m; o tempo de $0,30 \mathrm{~h}$ resultou no maior pico, de $63,10 \mathrm{~m}$; para o tempo de $0,10 \mathrm{~h}$, foi obtido o pico intermediário de 41,82m. Em seguida, foram gerados os mapas do indicador de velocidade (Figuras 4). 
Figura 4. Mapas de velocidade do escoamento para os tempos de formação de brecha. Tempo de formação de brecha de $0,10 \mathrm{~h}$ (A), tempo de formação de brecha de $0,30 \mathrm{~h}$ (B) e para o tempo de formação de brecha de $2,15 \mathrm{~h}$.
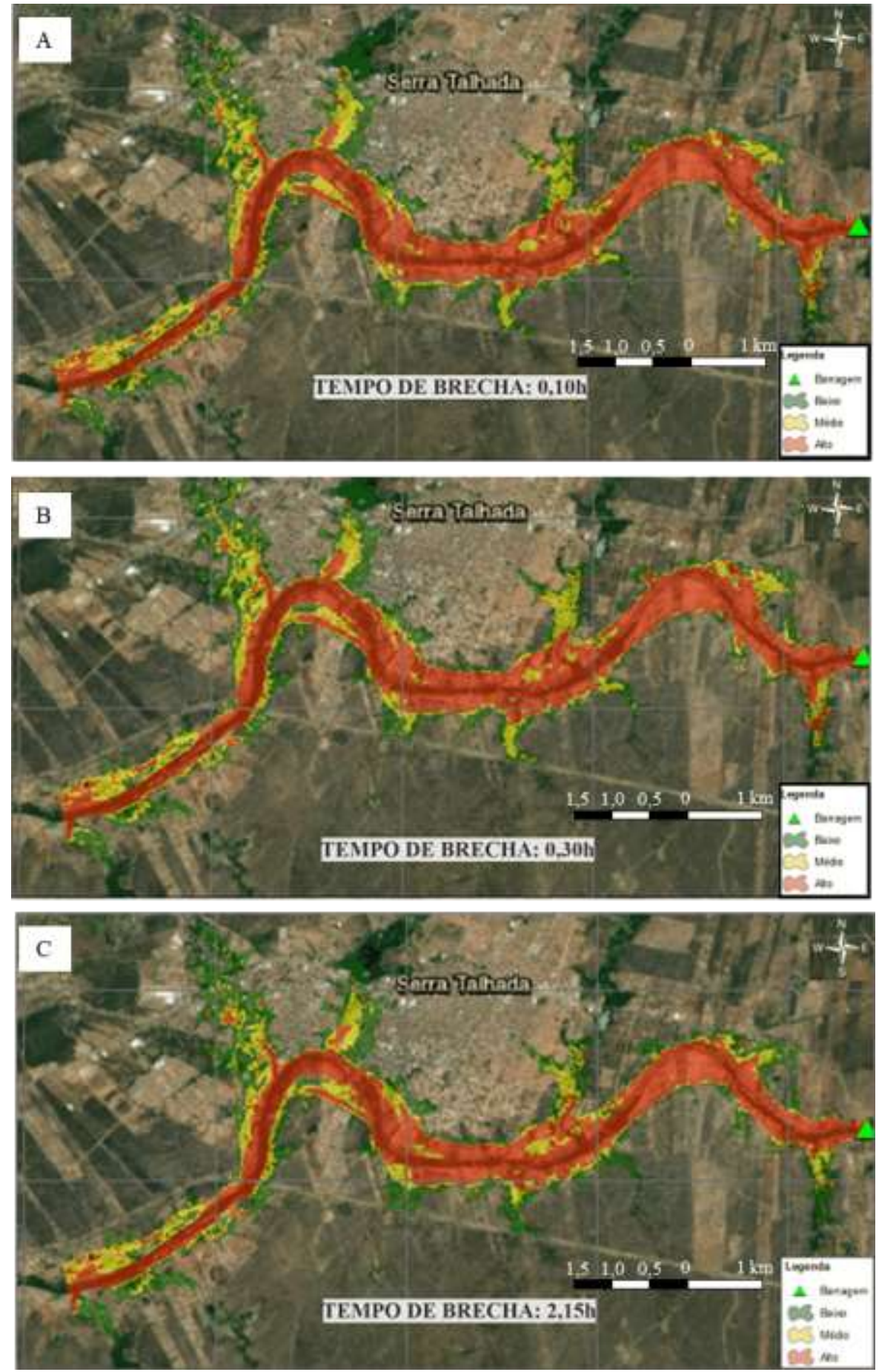

Fonte: Autores.

O indicador de velocidade (Figura 4) também apresenta, nos três tempos de formação de brecha estudados, uma maior concentração de área com nível de perigo alto. Embora em comparação com os outros dois indicadores analisados, o indicador de velocidade demonstrou uma maior área de nível de perigo baixo. Foram também gerados mapas de intensidade para os três tempos estudados (Figuras 5). 
Figura 5. Mapas de intensidade para os tempos de formação de brecha. Tempo de formação de brecha de $0,10 \mathrm{~h}$ (A), tempo de formação de brecha de $0,30 \mathrm{~h}$ (B) e para o tempo de formação de brecha de 2,15 h.
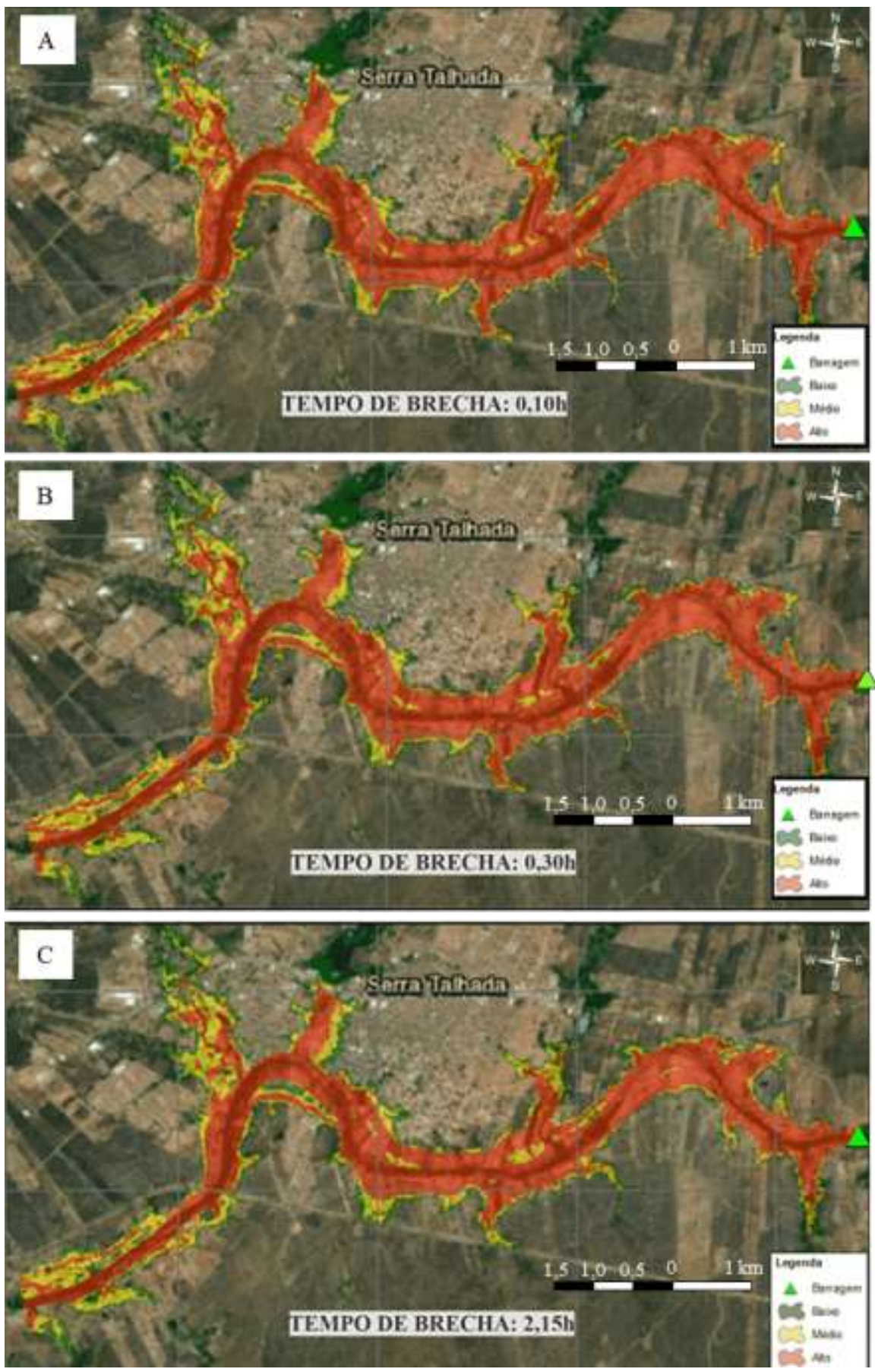

Fonte: Autores.

Os mapas de intensidade (Figura 5) também indicaram, em sua maior parte, valores classificados como alto. Também foram analisados três pontos no município de Serra Talhada-PE: a Secretaria Municipal de Obras e Infraestrutura, o Terminal Integrado de Transporte Alternativo, que são locais de apoio à população no caso de uma situação de risco de inundação, e a Rua Licácia Simonia de Souza, que está em um bairro com um grande número de residências e próximo ao curso d'água principal (Figura 6). 
Figura 6. Mapa de localização dos locais analisados no município de Serra Talhada-PE.

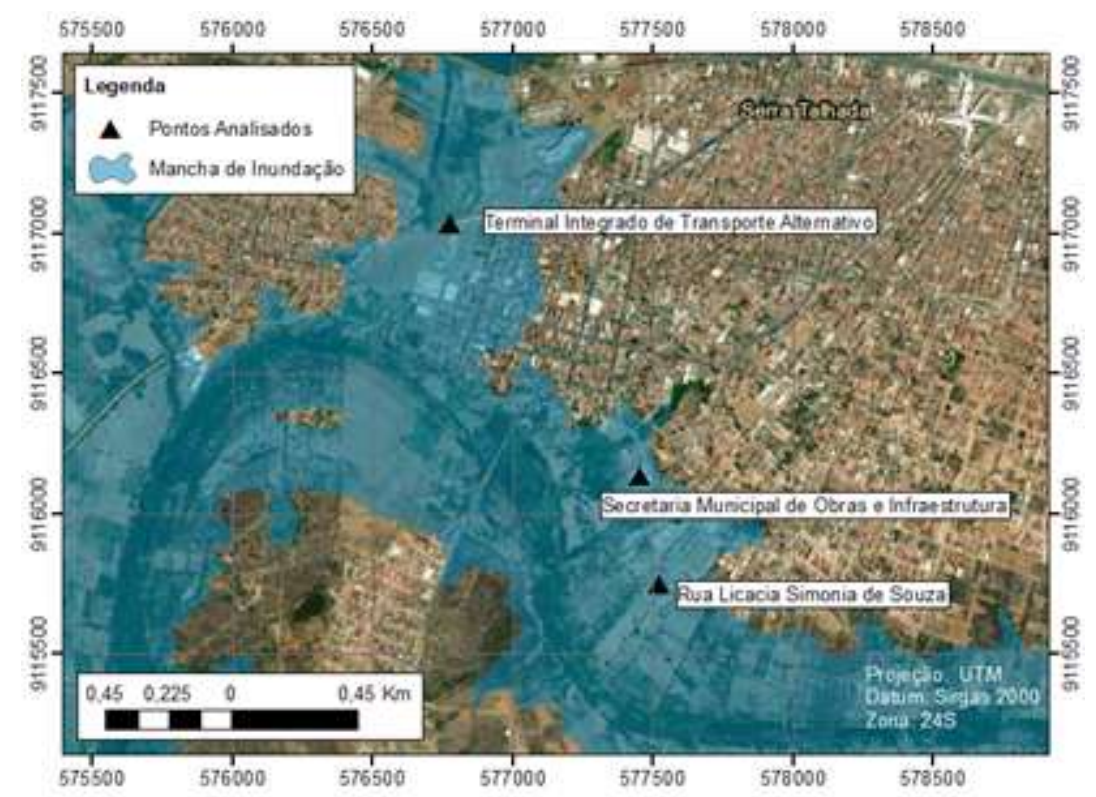

Fonte: Autores.

Os tempos de pico da altura do nível d'água para os tempos de formação de brecha de $0,10 \mathrm{~h}, 0,30 \mathrm{~h}$ e $2,15 \mathrm{~h}$ na Secretaria Municipal de Obras e Infraestrutura, foram de 1:40h, 1:45h e 3:05h, respectivamente (Figura 7). Para o Terminal Integrado de Transporte Alternativo, os tempos de pico foram, 1:50h., 2:00h e 3:15h, respectivamente (Figura 8). Já para a Rua Licácia Simonia de Souza, foram de 1:35h, 1:45h e 3:00h, respectivamente (Figura 9). Observou-se que os tempos de formação de brecha de $0,10 \mathrm{~h}$ e $0,30 \mathrm{~h}$ obtiveram tempos semelhantes de pico para todos os locais estudados.

Ademais, observou-se que apesar das distinções dos tempos de formação da brecha e dos tempos de pico, a altura máxima do nível d'água nas ondas de cheias foram idênticas em todos os tempos de brecha simulados em dois dos três locais estudados, a Secretaria Municipal de Obras e Infraestrutura (3,89m) e a Rua Licácia Simonia de Souza (2,00m). Porém, o Terminal Integrado de Transporte Alternativo chegou a obter mais de 1,00m de diferença entre os tempos de formação de brecha modelados, sua maior profundidade foi de 4,23m no tempo de formação de brecha de $0,30 \mathrm{~h}$ e sua menor profundidade foi de 3,15m no tempo de formação de brecha de 2,15h. Embora as profundidades nos locais estejam abaixo do maior pico de onda de cheia observado no mapa de perigo, ainda assim oferecem um nível alto de perigo à população.

Figura 7. Variação temporal da inundação na Secretaria Municipal de Obras e Infraestrutura.

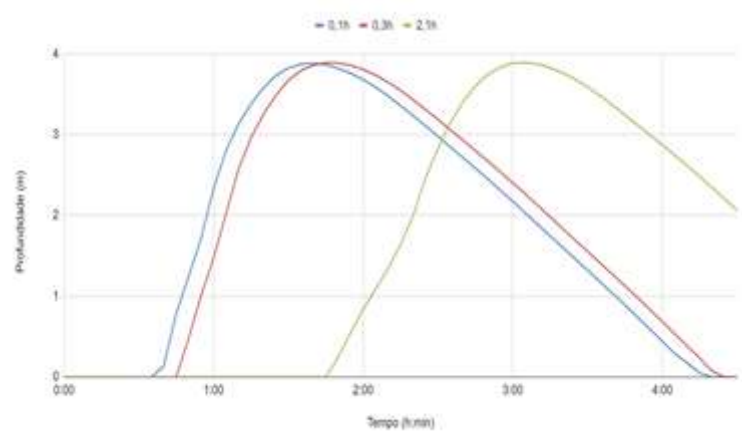

Fonte: Autores. 
Research, Society and Development, v. 10, n. 12, e572101220854, 2021

(CC BY 4.0) | ISSN 2525-3409 | DOI: http://dx.doi.org/10.33448/rsd-v10i12.20854

Figura 8. Variação temporal da inundação no Terminal Integrado de Transporte Alternativo.

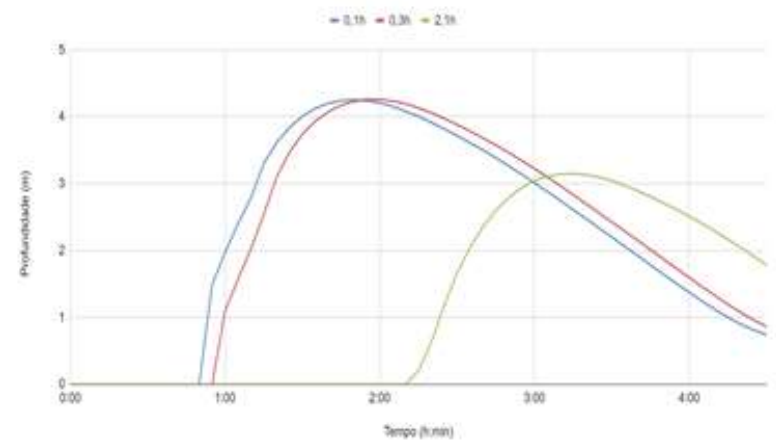

Fonte: Autores.

Figura 9. Variação temporal da inundação na Rua Licácia Simonia de Souza.

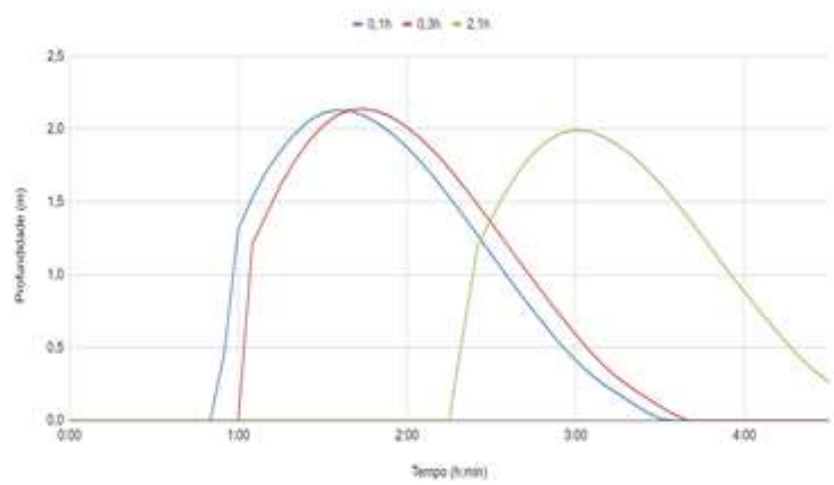

Fonte: Autores.

Por fim, apresenta-se uma tabela resumo, executada através da geração de seções transversais dos rasters obtidos, caracterizando a profundidade, elevação topográfica da lâmina d'água, vazão máxima e tempo de pico (Tabela 3 ). 
Tabela 3. Tabela resumo das seções transversais geradas.

\begin{tabular}{|c|c|c|c|c|c|}
\hline $\begin{array}{c}\text { Distância a } \\
\text { jusante (km) }\end{array}$ & $\begin{array}{c}\text { Cenário } \\
\text { (Tempo de } \\
\text { brecha) }\end{array}$ & $\begin{array}{c}\text { Profundidade máxima } \\
\text { na seção }(\mathbf{m})\end{array}$ & $\begin{array}{c}\text { Elevação } \\
\text { topográfica da } \\
\text { lâmina d'água } \\
\text { máxima (m) }\end{array}$ & $\begin{array}{l}\text { Vazão máxima } \\
\qquad\left(\mathbf{m}^{3} / \mathbf{s}\right)\end{array}$ & $\begin{array}{l}\text { Tempo de Pico } \\
\text { (HH:MM) }\end{array}$ \\
\hline \multirow[t]{3}{*}{0,300} & $0,10 h$ & 12,96 & 426,43 & 9994,17 & $00: 10$ \\
\hline & $\mathbf{0 , 3 0 h}$ & 13,01 & 426,48 & 9817,67 & 00:20 \\
\hline & $2,15 h$ & 12,17 & 425,61 & 7736,91 & 02:00 \\
\hline \multirow[t]{3}{*}{6,530} & $0,10 h$ & 10,49 & 419,31 & 5795,57 & 01:10 \\
\hline & $0,30 h$ & 10,50 & 419,32 & 5822,00 & 01:20 \\
\hline & $2,15 h$ & 10,33 & 419,14 & 5411,42 & $02: 40$ \\
\hline \multirow[t]{3}{*}{11,250} & $0,10 h$ & 9,42 & 416,43 & 3694,66 & 01:55 \\
\hline & $\mathbf{0 , 3 0 h}$ & 9,41 & 416,44 & 3701,37 & 02:00 \\
\hline & $2,15 h$ & 9,41 & 416,31 & 3541,99 & 03:20 \\
\hline \multirow[t]{3}{*}{14,100} & $0,10 h$ & 7,98 & 412,84 & 3532,33 & 02:20 \\
\hline & $\mathbf{0 , 3 0 h}$ & 8,94 & 412,84 & 3537,30 & $02: 25$ \\
\hline & $2,15 h$ & 8,94 & 412,75 & 3393,15 & $03: 45$ \\
\hline
\end{tabular}

Fonte: Autores.

Notou-se que as variações dos picos de vazão para diferentes cenários mostraram-se mais significativas nas seções mais próximas ao barramento. Enquanto para $0,30 \mathrm{~km}$ houve uma redução de $22,60 \%$ quando utilizado o maior tempo de brecha, para 14,10km a redução da vazão foi de apenas 4,10\%. Embora os picos de vazão para grandes distâncias sejam próximos, a diferença entre tempos de pico ainda é alta $(01 \mathrm{~h} 25 \mathrm{~min})$. As variações entre os tempos de brecha propostos pela ANA (2016) mostraram-se insignificantes. As vazões máximas para os cenários de $0,10 \mathrm{~h}$ e $0,30 \mathrm{~h}$ apresentaram variações de $1,80 \%$ a $0,10 \%$ à medida que a seção se distancia do barramento. Da mesma forma, repercute em uma variação ainda menor em termos de elevação topográfica, apresentando percentuais relativos inferiores a $0,10 \%$.

\section{Considerações Finais}

Conforme apresentado neste estudo, a ruptura hipotética da barragem Jazigo na cidade de Serra Talhada em Pernambuco leva a uma cheia que inundaria regiões urbanas do município, afetando equipamentos urbanos importantes, com a maior parte da área inundada apresentando um indicador de intensidade de grande potencial de dano à população. Mesmo utilizando três diferentes tempos de formação de brecha, os mapas apresentam grandes semelhanças nas variáveis analisadas, sempre com a maior parte da região atingida na faixa de valores classificados como alto. Também foi constatado que o hidrograma de cheia de ruptura que possui uma maior translação do tempo de pico e amortecimento da descarga máxima foi o obtido com o tempo de brecha da equação de Froehlich. Nos outros dois tempos recomendados pela ANA, os hidrogramas se 
comportaram de forma semelhante, com uma pequena translação para a direita do com tempo de brecha de 0,30h. Ainda, constatou-se pequena variação nos resultados ao escolher entre os tempos de brecha propostos pela ANA, demonstrando que adotar $0,10 \mathrm{~h}$ ou $0,30 \mathrm{~h}$ não impactou substancialmente na caracterização das áreas de risco neste caso. Por outro lado, a adoção do tempo de brecha através da equação de Froehlich mostrou maiores picos em regiões próximas ao barramento e tempos de pico mais longos, mesmo em regiões mais distantes da barragem, quando comparados com os limites recomendados no manual da ANA. Espera-se, com esse estudo, contribuir para a adoção de medidas de planejamento urbano das áreas atingidas e adoção de medidas estruturais e não estruturais para a cidade de Serra Talhada, em busca de diminuir os danos sociais, econômicos e ambientais causados em caso de uma eventual ruptura.

Trabalhos futuros podem avaliar se os resultados da inundação mantêm o mesmo comportamento para casos em barragens de grande porte e para extensões maiores de vale à jusante. Outra possibilidade é analisar a sensibilidade geral dos tempos de brecha através de métodos não físicos ou estatísticos. Por fim, ainda sugere-se estudar como os demais parâmetros de brecha, a exemplo de largura média e inclinação, impactam nos valores de classificação de risco de cheia.

\section{Referências}

Agência Nacional de Águas e Saneamento Básico. (2016). Manual do Empreendedor sobre Segurança de Barragens: Guia de Orientação e Formulário do Plano de Ação de Emergência (PAE). Brasília-DF, 127 p.

Balogun, O. e Ganiyu, H. (2017). Development of Inundation Map for Hypothetical Asa Dam Break using HEC-RAS and ARC GIS. Arid Zone Journal of Engineering, Technology and Environment, 13, 831-839.

Banco mundial. (2014). "Serviços Analíticos e Consultivos em Segurança de Barragens: Produto 4 - Classificação de Barragens: Avaliação dos Critérios Gerais Atuais, Metodologia Simplificada para Áreas Inundadas a Jusante e Diretrizes para a Classificação.” 78 p.

Brunner, Gary W. (2016). HEC-RAS, River Analysis System Hydraulic Reference Manual. U.S. Army Corps of Engineers Hydrologic Engineering Center (HEC), Davis-CA. 547p.

Dias, G. G. (2011). Curso Segurança de Barragens: Módulo III - Gestão e desempenho de Barragens - Unidade 4: Relatórios de Inspeção. Material produzido no âmbito do Convênio $n^{\circ}$ 001/ANA/2011 - SICONV no 756001/2011, firmado entre a Agência Nacional de Águas - ANA e a Fundação Parque Tecnológico de Itaipu - Brasil - FPTI. $31 \mathrm{p}$.

Faria, F. L.F., B., Silva, M., de M. Reis, M., e Amorim, J. C. C. (2019). Metodologia para obtenção do hidrograma para simulação de ruptura de barragens. Revista Militar De Ciência E Tecnologia, 36(3).

Froehlich, D.C.; Ph.D.; P.E., D.WRE, M.ASCE. (2016). Empirical model of embankment dam breaching. Journal of Hydrologic Engineering, 21(11).

Governo do Estado de Pernambuco (2016). Pernambuco Tridimensional. http://www.pe3d.pe.gov.br/.

Guo, Kaihua, Guan, Mingfu e Yu, Dapeng. (2021). Urban surface flood modelling - a comprehensive review of current models and future challenges. Hydrology and Earth System Sciences (Hydrol. Earth Syst. Sci.), 25, 2843-2860.

Instituto Brasileiro de Geografia e Estatística. (2020). Cidades: panorama. "Serra Talhada". https://cidades.ibge.gov.br/brasil/pe/serra-talhada/panorama . Acesso em 28 de abr. de 2021.

International Commission on Large Dams (2019). The History of the World Register of Dams. www.icold-cigb.org/GB/world_register/history.asp. Acesso em 2 ago. de 2021 .

Justiça Federal da $5^{\circ}$ Região (2014). www.jfpe.jus.br/index.php/institucional/biblioteca/761-historico-do-municipio-serra-talhada.html. Acesso em 2 ago. de 2021 .

Lei Federal $n^{\circ}$ 12.334, de 20 de setembro de 2010 (2010). Estabelece a Política Nacional de Segurança de Barragens destinadas à acumulação de água para quaisquer usos, à disposição final ou temporária de rejeitos e à acumulação de resíduos industriais, cria o Sistema Nacional de Informações sobre Segurança de Barragens e altera a redação do art. 35 da Lei n 9.433, de 8 de janeiro de 1997, e do art. $4^{\circ}$ da Lei no 9.984, de 17 de julho de 2000 ”. Diário Oficial da União, Brasília-DF.

Lei Federal $n^{\circ}$ 14.066, de 30 de setembro de 2020 (2020). Altera a Lei no 12.334, de 20 de setembro de 2010, que estabelece a Política Nacional de Segurança de Barragens (PNSB), a Lei n ${ }^{\circ} 7.797$, de 10 de julho de 1989, que cria o Fundo Nacional do Meio Ambiente (FNMA), a Lei nº.433, de 8 de janeiro de 1997, que institui a Política Nacional de Recursos Hídricos, e o Decreto-Lei n 227, de 28 de fevereiro de 1967 (Código de Mineração)”. Diário Oficial da União, Brasília-DF.

Mello, F.M. A história das barragens no Brasil, Séculos XIX, XX e XXI (2011). Comitê Brasileiro de Barragens.

Neves, Y. T., Rodrigues, A. B. e Cabral, J. J. S. P. (2021). Modelagem computacional do rompimento hipotético da barragem de Jucazinho no estado de Pernambuco (Brasil). Revista DAE. 69. 167-182. 
Pereira A. S. et al. (2018). Metodologia da pesquisa científica. UFSM.

Prieto Calderon, J.L., Marínez-Alegría-López, R., Taboa J., Montequi, I. e Sanz, G. (2017). Rotura de la presa de Vega de Tera, simulación hidráulica de la propagación de la avenida (Zamora, España). DYNA, 84(203), 45-54.

Ribeiro Neto, A., Batista, L.F. D. R. e Coutinho, R.(2016). "Q.1. Methodologies for generation of hazard indicator maps and flood prone areas: municipality of Ipojuca/PE”. RBRH, 21(2), 377-390.

Rocha, A. K. P. (2018). Trabalho de conclusão de curso (TCC) em Engenharia Civil. UFRPE.

Sabău, D. A. e ŞERBAN, G. (2018). Arch dam failure preliminary analysis using HEC-RAS and HEC-GEO RAS modeling. Case study Someșul Rece 1 reservoir. Forum geografic. XVII. 44-55.

Secretaria de Infraestrutura e Recursos Hídricos (2020). “Relatório de Inspeção de Segurança Regular - Barragem Jazigo”.

Silva, B. M. e Montenegro, S. G. L. (2009). Chuvas intensas em localidades do Estado de Pernambuco. Dissertação (Mestrado) - Programa de Pós-Graduação em Engenharia Civil. Universidade Federal de Pernambuco. 116 p.

Souza, T. S. A., Ferreira, L. D. e Oliveri, A. M. R. (2019). Evolução histórica da legislação brasileira e do estado de Minas Gerais relacionado ao tema de disposição de rejeitos de mineração em barragens. 2019. 93 f. Monografia (Graduação em Engenharia Civil) - Escola de Minas, Universidade Federal de Ouro Preto, Ouro Preto.

USACE (2014). United States Army Corps of Engineers. Using HEC-RAS for dam break studies. USACE. 74 p.

U.S. Department of the interior, burreu of reclamation engineering (Estados Unidos). Policy and Procedures for Dam Safety Modification Decision-making. Denver: U.S. Department of the Interior, Bureau of Reclamation Engineering, 1989. Página III-13.

Wahl, T. (2004). Uncertainty of predictions of embankment dam breach parameters. Journal of Hydraulic Engineering, $130,389-397$.

Yin, R.K. (2015). O estudo de caso. Bookman. 